\title{
DNA Damage Recognition of Mutated Forms of UvrB Proteins in Nucleotide Excision Repair
}

\author{
Yue Zou ${ }^{*}$, , Huaxian Ma $\ddagger$, Steven M. Shell $\ddagger$, and Zhengguan Yang $\ddagger$ \\ Department of Biochemistry and Molecular Biology, James H. Quillen College of Medicine, East \\ Tennessee State University, Johnson City, Tennessee 37614, \\ R. Stephen Lloyd§ and Irina G. Minko§ \\ Sealy Center for Molecular Science, University of Texas Medical Branch, Galveston, Texas 77555, \\ Youxing Qull and Ying Xull \\ Protein Informatics Group, Life Sciences Division, Oak Ridge National Laboratory, Oak Ridge, \\ Tennessee 37830 \\ Nicholas E. Geacintov ${ }^{\perp}$ \\ Department of Chemistry, New York University, New York, New York 10003
}

\begin{abstract}
The DNA repair protein UvrB plays an indispensable role in the stepwise and sequential damage recognition of nucleotide excision repair in Escherichia coli. Our previous studies suggested that UvrB is responsible for the chemical damage recognition only upon a strand opening mediated by UvrA. Difficulties were encountered in studying the direct interaction of UvrB with adducts due to the presence of UvrA. We report herein that a single point mutation of Y95W in which a tyrosine is replaced by a tryptophan results in an UvrB mutant that is capable of efficiently binding to structurespecific DNA adducts even in the absence of UvrA. This mutant is fully functional in the UvrABC incisions. The dissociation constant for the mutant-DNA adduct interaction was less than $100 \mathrm{nM}$ at physiological temperatures as determined by fluorescence spectroscopy. In contrast, similar substitutions at other residues in the $\beta$-hairpin with tryptophan or phenylalanine do not confer UvrB such binding ability. Homology modeling of the structure of $E$. coli UvrB shows that the aromatic ring of residue Y95 and only Y95 directly points into the DNA binding cleft. We have also examined UvrB recognition of both "normal" bulky BPDE-DNA and protein-cross-linked DNA (DPC) adducts and the roles of aromatic residues of the $\beta$-hairpin in the recognition of these lesions. A mutation of Y92W resulted in an obvious decrease in the efficiency of UvrABC incisions of normal adducts, while the incision of the DPC adduct is dramatically increased. Our results suggest that Y92 may function differently with these two types of adducts, while the Y95 residue plays an unique role in stabilizing the interaction of UvrB with DNA damage, most likely by a hydrophobic stacking.
\end{abstract}

It is generally believed that the broad spectrum of DNA substrates of nucleotide excision repair $(\mathrm{NER})^{1}$ is secured by its unique machinery of damage recognition (1-3). Such a wide range of damage recognition was recently further expanded to include a DNA-protein cross-link as a substrate of NER (4). Understanding of this recognition process includes the structural determination and the characterization of the biochemical interactions between proteins and adducts and is a crucial step toward defining the molecular mechanism of NER. In Escherichia

\footnotetext{
*To whom correspondence should be addressed. Phone: (423) 439-2124. Fax: (423) 439-2030. E-mail: zouy@etsu.edu.

East Tennessee State University.

\$University of Texas Medical Branch.

"Oak Ridge National Laboratory.

$\perp_{\text {New York University. }}$
} 
coli NER, the DNA damage recognition occurs through a sequential two-step mechanism in which the adduct-induced disruption of the Watson-Crick DNA structure is recognized at the initial step most likely by $\mathrm{UvrA}_{2}$ of an $\mathrm{UvrA}_{2} \mathrm{~B}$ complex, while the type of modifications of the nucleotide is recognized at the following step upon strand opening $(5,6)$. The UvrB protein, a central player in the UvrABC nuclease system of E. coli NER, is directly involved in the second step of damage recognition in addition to its crucial roles in strand opening, recruitment of $\mathrm{UvrC}$, and subsequent incisions. The indispensable role of UvrB in the recognition of the modified nucleotide was also demonstrated by the efficient UvrBC incisions of adducts in a largely opened DNA structure even in the absence of UvrA $(5,6)$.

Recent determinations of crystal structures of UvrB proteins from Bacillus caldotenax (7) and Thermus thermophilus $(8,9)$ revealed a core structure of helicases featured with a flexible $\beta$ hairpin which is essential for DNA binding and damage processing. Subsequent functional studies of UvrB with mutations in the $\beta$-hairpin region demonstrated the importance of this hairpin motif in DNA binding and damage recognition $(10,11)$. Several hydrophobic residues in the motif thereafter have been shown to be crucial for damage recognition and specificity (10). However, the question of how UvrB interacts with such a large diversity of adducts, as well as the biochemical basis and characteristics of the recognition, remains to be addressed. Understanding of these problems requires the study of direct interactions of UvrB with the adducts. The major hurdle to such efforts was that, in the UvrABC system, binding of UvrB to the adduct is mediated by the UvrA which loads the protein to the damaged site. In the absence of UvrA, UvrB is unable to bind or recognize DNA or DNA damage directly. In this respect, the development of a system with the UvrB protein capable of binding directly to DNA damage is important and would provide further insights into the mechanism of UvrB binding.

On the other hand, we recently reported that NER, as a potential repair pathway, is also involved in the repair of DNA-protein (T4-pdg) cross-links (DPC) although this type of DNA adduct is structurally very different from the normal bulky adducts removed by NER (4). Since UvrB may play a key role in the recognition of DPC, the interesting question needs to be addressed whether the same molecular interactions of the amino acid residues of UvrB with normal adducts also occur in the case of the recognition of the highly bulky DPC proteins. Considering such great structural diversity of substrates of NER, the aim of this investigation is to provide a better understanding of the mechanism of damage recognition by NER.

In the present study, we demonstrate that a single mutation of Y95W strikingly enhances the binding of UvrB to structure-specific DNA adducts without the participation of UvrA, while

\author{
${ }^{1}$ Abbreviations: \\ BPDE \\ benzo $[a]$ pyrene diol epoxide or 7,8-dihydroxy-9,10-epoxy-7,8,9,10-tetrahydrobenzo[ $[a]$ pyrene \\ CPD \\ cyclobutane pyrimidine dimer \\ DPXL \\ DNA-protein cross-linking \\ DTT \\ dithiothreitol \\ EDTA \\ ethylenediaminetetraacetic acid \\ F-DNA \\ fluorescein-DNA \\ NER \\ nucleotide excision repair.
}


mutations at other nearby aromatic and hydrophobic sites in the $\beta$-hairpin did not exhibit any enhancement in binding. Furthermore, the Y95W mutant is fully functional in the UvrABC incision assay. The presence of the tryptophan also renders the UvrB fluorescent. Using fluorescence spectroscopy, the binding constant was determined for damage recognition by UvrB. Differences and similarities in the profiles of mutation-function relationships of the $\beta$ hairpin residues were observed with the normal bulky DNA adducts and the DPC adduct. In particular, the Y92W UvrB mutant showed a significant enhancement in the UvrABC incision efficiency of the DPC adduct in comparison with UvrABC incision with the native UvrB protein; however, such an effect was not observed in the case of the normal adducts, suggesting a specific role of this residue in the interaction with DPC adducts. Our mutant systems also provide new opportunities for future more biochemically oriented investigations of NER damage recognition, including cocrystal structural determinations of UvrB-DNA adduct interactions.

\section{EXPERIMENTAL PROCEDURES}

DNA Substrate Construction. The $50 \mathrm{bp}$ DNA substrates used for this study were constructed as described previously (6). To make the $30 \mathrm{bp}$ substrates, phosphorylated 11 mers ( $30 \mathrm{pmol}$ ) containing a single (+)-cis-anti-BPDE adduct or a cyclobutane pyrimidine dimer (CPD) adduct were ligated with stoichiometric quantities of phosphorylated 19mer, using T4 DNA ligase in the presence of a 35 mer template strand containing the complementary sequence in a $30 \mu \mathrm{L}$ solution containing $50 \mathrm{mM}$ Tris- $\mathrm{HCl}, \mathrm{pH} 7.8,10 \mathrm{mM} \mathrm{MgCl}_{2}, 10 \mathrm{mM}$ DTT, $1 \mathrm{mM}$ ATP, and 50 $\mu \mathrm{g} / \mathrm{mL}$ of BSA. The ligation was carried out at $16^{\circ} \mathrm{C}$ for $12 \mathrm{~h}$. After ligation, the products were purified and then reannealed with a partially complementary 30mer template strand to make appropriate bubble substrates, as shown in Figure 1. The annealed substrates were purified on a nondenaturing $8 \%$ polyacrylamide gel. The double-stranded character and homogeneity of the substrates were examined by a restriction assay (12). The fluorescein-30 bp bubble DNA substrate was obtained by annealing a synthesized oligonucleotide 30mer containing a fluorescein to a partially complementary oligonucleotide strand (Figure 1). All oligonucleotides used for substrate construction were purified by PAGE under denaturing conditions.

The DNA-protein cross-link substrate (DPC) was prepared as described previously (4).

Protein Production and Purification. UvrB mutants were overproduced and purified in one step through a chitin column from $E$. coli C41(DE3) cells (23) harboring the plasmids containing the appropriate mutant $U v r B$ genes, which were generated from site-directed mutagenesis of the construct pUTG97 (6) using the QuikChange kit (Stratagene). UvrA and UvrC proteins were purified as reported previously (6). Protein concentrations were determined by the Bio-Rad protein assay.

Gel Mobility Shift Assay. Binding of the DNA substrates by the Uvr proteins was determined by gel mobility shift assays. Typically, the substrate $(3 \mathrm{nM})$ was incubated with UvrA $(15 \mathrm{nM})$ and/or UvrB or UvrB mutants at the indicated concentrations at $37^{\circ} \mathrm{C}$ for $15 \mathrm{~min}$ in $20 \mu \mathrm{L}$ of UvrABC buffer (50 mM Tris-HCl, pH 7.5, $50 \mathrm{mM} \mathrm{KCl,} 10 \mathrm{mM} \mathrm{MgCl}_{2}, 5 \mathrm{mM}$ DTT) in the presence or absence of $1 \mathrm{mM}$ ATP. After incubation, $2 \mu \mathrm{L}$ of $80 \%(\mathrm{v} / \mathrm{v})$ glycerol was added, and the mixture was immediately loaded onto a $3.5 \%$ native polyacrylamide gel in TBE running buffer and electrophoresed at room temperature. For the binding studies with both proteins UvrA and $\mathrm{UvrB}$, ATP $(1 \mathrm{mM})$ and $\mathrm{MgCl}_{2}(10 \mathrm{mM})$ were present in the TBE running buffer.

UvrABC Incision Assay. The 5'-terminally labeled DNA substrates $(3 \mathrm{nM})$ were incised by UvrABC or UvrBC (UvrA, $15 \mathrm{nM}, \mathrm{UvrB}, 250 \mathrm{nM}$, and UvrC, $50 \mathrm{nM}$ ) in the UvrABC buffer $(1 \mathrm{mM} \mathrm{ATP})$ at $37^{\circ} \mathrm{C}$ for $30 \mathrm{~min}$. For the incision kinetics measurement, the substrates were 
incised for $0,5,10,20$, and $30 \mathrm{~min}$. The Uvr subunits were diluted and premixed into storage buffer before being mixed with DNA. The reactions were terminated by adding EDTA (20 $\mathrm{mM}$ ) or heating to $90^{\circ} \mathrm{C}$ for $3 \mathrm{~min}$. The samples were denatured with formamide and heated to $90{ }^{\circ} \mathrm{C}$ for $5 \mathrm{~min}$ and then quick-chilled on ice. The digested products were analyzed by electrophoresis on a $12 \%$ polyacrylamide sequencing gel under denaturing conditions with TBE buffer.

Fluorescence Spectroscopy Determination. The tryptophan fluorescence spectra of UvrB mutants were recorded at $25{ }^{\circ} \mathrm{C}$ on a SPEX Fluorolog-3 fluorometer with the excitation wavelength at $295 \mathrm{~nm}$ and the slit widths set at $3 \mathrm{~nm}$ for excitation and $5 \mathrm{~nm}$ for emission beams. The binding of the $\mathrm{UvrB}(\mathrm{Y} 95 \mathrm{~W})$ mutant to the DNA substrate containing a fluorescein was measured by monitoring the emission of fluorescein at $\left.520 \mathrm{~nm}\left(\gamma_{\mathrm{ex}}\right) 492 \mathrm{~nm}\right)$ at $37{ }^{\circ} \mathrm{C}$ in the UvrABC buffer in the absence of ATP. The slit widths were set at $1 \mathrm{~nm}$ for excitation and $5 \mathrm{~nm}$ for emission beams. The titrations were performed in a micro quartz cuvette for a minimum sample volume of $200 \mu \mathrm{L}$ with a $2 \mathrm{~mm} 2 \mathrm{~mm}$ stirring bar. In these experiments, the $\mathrm{UvrB}(\mathrm{Y} 95 \mathrm{~W}) \times$ and the DNA substrate were placed in the same binding buffer before the titration, so that the background did not change during the addition of protein. Each addition of $\mathrm{UvrB}(\mathrm{Y} 95 \mathrm{~W})$ protein was $0.5-1 \mu \mathrm{L}$, delivered by a $25 \mathrm{uL}$ Hamilton syringe using a Hamilton repeating dispenser.

Protein Structure Modeling. The structures of wild-type E. coli UvrB and its mutants Y95F, Y95W, and Y96W were predicted by homology modeling based on the $2.6 \AA$ crystal structure of B. caldotenax $\mathrm{UvrB}$ (PDB code $1 \mathrm{~d} 9 \mathrm{x}$ from ref 7). The sequence alignment profile was also taken from Theis et al. (7), which reported the multiple alignment of UvrB sequences from three different species (B. caldotenax, T. thermophilus, and E. coli) with the programs ClustalW and ALSCRIPT. The sequence identity between UvrB from B. caldotenax and E. coli ( $T$.

thermophilus) is 59\% (60\%). Homology modeling and optimization of $E$. coli UvrB structures were carried out using the program MODELLER (13). The quality of the modeled structures was evaluated by the PROCHECK (14) program. Approximately $98 \%$ of the residues are in the most favored and the additionally allowed regions of the Ramachandran plot, and only $1 \%$ of the residues [V195, R196, E282, D481, V221 (Y95F only), V408 (Y96W only)] are in the disallowed region.

\section{RESULTS}

Construction of DNA Substrates. The structures of DNA adducts and substrates used in this study are presented in Figure 1. Structurally, there are three different types of DNA substrates. The site-specific DNA-protein cross-link substrate $(60 \mathrm{bp})$ was built from the catalytic chemistry of the T4 pyrimidine dimer glycosylase/apurinic/apyrimidinic site lyase (T4-pdg) (4). As a control, a substrate of the same DNA sequence with the substitution of the T4-pdg by a uracil was also constructed. The 50 bp oligodeoxynucleotide substrates with both bubble and nonbubble structures contained an adduct (BPDE) in the middle of the top strand (Figure 1). The BPDE was adducted at the $\mathrm{N} 2$ position of the guanine. The $30 \mathrm{bp}$ substrates were constructed with an adduct asymmetrically located in the top strand and five nucleotides $5^{\prime}$ to the adduct.

Site-Specific Mutagenesis of UvrB. On the basis of the determination of the crystal structure, it has been suggested that the $\beta$-hairpin of the UvrB protein plays an important role in damage recognition by UvrB (7). Replacement of hydrophobic residue pairs in the $\beta$-hairpin region with alanines suggested that these amino acids are involved in the damage recognition (10). To determine the biochemical characteristics and nature of the interactions of UvrB with adducted DNA, we substituted the individual hydrophobic residues of UvrB with tryptophan. The purposes of the site-directed mutagenesis were severalfold: to identify individual residues 
involved in the recognition of normal bulky adducts or DPC, to probe the nature of the specific interactions and their contributions to damage recognition, to enhance and manifest the intrinsic affinity of UvrB for adducts which is generally undetectable in the absence of UvrA, and to introduce a fluorophore into the nonfluorescent UvrB protein for further biochemical studies. The choice of tryptophan in the mutation studies was obvious due to its fluorescence and its hydrophobic characters. The tryptophan residue is also aromatic, with a structure resembling that of a nucleobase.

UvrABC Incisions of Normal Bulky Adducts and the DPC Adduct with UvrB Mutants. As shown in Figure 2A,B, the 50 bp DNA substrate containing a single (+)-cis-BPDE-dG adduct was incised by UvrABC nuclease with wild-type UvrB or mutants in a kinetic assay. The substrate was labeled at the 5'-end of the modified strand. The results demonstrated that all of these mutants are functionally effective in the recognition and incision of adducts but with varying efficiencies. In each case, the $5^{\prime}$-incisions occurred at the same eighth phosphate $5^{\prime}$ to the modified guanine. The initial rates of the incision were determined by a linear least-squares fitting of the data points collected over a defined incision period. The incision efficiencies for the mutants as compared to that of the wild-type follow the order of WT $\approx \mathrm{Y} 95 \mathrm{~W}>\mathrm{Y} 101 \mathrm{~W}>$ $\mathrm{Y} 92 \mathrm{~W}$. Specifically, the Y95W mutant resulted in an incision efficiency of $0.21(0.03 \mathrm{fmol} /$ min which was equivalent to that $(0.22(0.02 \mathrm{fmol} / \mathrm{min})$ for wild-type UvrB, while the efficiency of $\mathrm{Y} 101 \mathrm{~W}$ was about $0.16(0.02 \mathrm{fmol} / \mathrm{min}$. In contrast, the adduct was incised significantly more slowly by the $\operatorname{UvaAB}(\mathrm{Y} 92 \mathrm{~W}) \mathrm{C}$ than by the native proteins $(0.084(0.011$ $\mathrm{fmol} / \mathrm{min}$ ), suggesting the potential involvement of the Y92 residue in the formation of the UvrBC-DNA preincision intermediate. Similar results were also obtained with other types of adducts such as fluorescein- and AAF-DNA.

Despite the much larger molecular size of the protein cross-linked with the DNA in the DPC complex as compared to the normal bulky adduct (e.g., BPDE-dG), the DNA-protein (T4-pdg) cross-link was recently found to be a good substrate of the E. coli nucleotide excision repair apparatus (4). Considering the significant differences in the structure between the BPDE-dG lesions and the DPC adduct, it is of great interest to examine and compare the effects of UvrB mutations on the incisions of these two types of adducts. Therefore, similar incision experiments were performed with the substrate containing a single DNA-protein (T4-pdg) cross-link adduct (Figure 2C). The DPC substrate was labeled at the 5'-ends of both strands for the purpose of quantification. The migration of the protein-cross-linked DNA strand in a $12 \%$ polyacrylamide denaturing gel was so slow that the DPC strand remained very close to the bottom of the loading wells. Interestingly, in contrast to the results with the BPDE-DNA adduct, the incision efficiency of DPC with the Y92W mutant is greater than with the wildtype UvrB and the other mutants including Y95W and Y101W UvrB. Kinetic determinations indicated that the incision rate with $\mathrm{Y} 92 \mathrm{~W}$ is $0.078(0.016 \mathrm{fmol} / \mathrm{min}$, which is at least 2-fold more efficient than the others $(0.038(0.01,0.03(0.01$, and $0.026(0.008 \mathrm{fmol} / \mathrm{min}$ for wildtype, Y95W, and Y101W UvrB, respectively). The incisions are significantly different as the $p$-values from the $t$-test of incision comparison of Y92W with wild-type, Y95W, and $\mathrm{Y} 101 \mathrm{~W}$ are $0.024,0.012$, and 0.007 (all $<0.05$ ), respectively. The differences in incision profiles between the normal bulky and the DPC adducts imply that the influence of some of these hydrophobic residues on damage recognition and incision by UvrABC nuclease is different in these two cases and that the effect of the Y92 residue depends on the type of adduct.

Binding of UvrB and Mutants to DNA Substrates. To explore the potential intrinsic affinity of UvrB for DNA damage, we have purified several UvrB mutants. These mutants together with the native UvrB were then examined for DNA binding in the gel mobility shift assays as shown in Figure 3. To enhance the binding, structure-specific DNA bubble substrates were constructed (Figure 1) and adopted in the assays. As shown in Figure 3A, among all the mutants and the native UvrB, only the Y95W mutant showed substantially strong affinity for the adducts, 
indicating that the substitution of the Y95 residue by a tryptophan converted UvrB to a standalone DNA binding protein that manifested its intrinsic damage recognition power. The same results were obtained with both the aromatic adducts, BPDE and fluorescein. It is especially interesting that the Y96W protein was unable to bind to the DNA substrate albeit the residue was just next to the Y95W, suggesting a unique role of this residue in the interaction with the lesions. In addition, a longer exposure of the gel to the X-ray film or Phosphorimager screen showed a faint band for the formation of Y95F-DNA complexes. The order of the affinity for DNA substrates was Y95W. Y95F > WT, which was a strong indication of the involvement of the hydrophobic nature of these interactions.

While the 30 bp bubble substrate with an adduct asymmetrically located in the sequence (Figure 1) was efficiently recognized by the Y95W mutant, the binding of the mutant protein to the 50 bp DNA containing a single adduct in a bubble region in the middle of the top strand (Figure 1) was also equally efficient (Figure $3 \mathrm{~A}$ ). In contrast, the Y95W mutant showed no affinity for a 50 bp nonbubble substrate containing a BPDE-DNA adduct in the middle (data not shown). The Y95W protein was also tested for binding with nonbubble substrate as compared to the bubble substrates, and like the native UvrB, the mutant showed no affinity for the nonbubble DNA adduct (Figure 3A). Similar experiments were also conducted with a BPDE-singlestranded DNA 30mer, and no binding was evident (data not shown).

To understand the nature of the interactions of UvrB with adducts, the UvrB and mutants were also subjected to binding to a nonaromatic adduct, the cyclobutane pyrimidine dimer (CPD). As shown in Figure 3B, similar to the case of the aromatic adducts described above, the substrate was efficiently recognized only by the Y95W mutant but not by the others. Since the pyrimidine dimer adduct formed between the intrinsic two thymine bases without exogenous molecules is a good substrate for UvrABC nuclease (15), it is reasonable to assume that the protein may also bind to nondamaged DNA bubble substrates. To test this possibility, similar bubble substrates containing no adduct were con-structed and used in the binding assay with the Y95W mutant. The results indicated that the protein did bind to the nondamaged DNA although the affinity was lower than for damaged substrates (Figure 3B). However, the binding to nondamaged substrate was reduced in the presence of ATP and was completely eliminated in the presence of both UvrA and ATP (data not shown). It is evident that the binding of Y95W to nondamaged DNA is weak under the conditions with ATP, which is indispensable for a successful NER. These results strongly suggest that the Y95 residue of UvrB is directly involved in the interaction with the adducts and that it is facilitated at the same time by interactions with neighboring DNA bases. The present results are also consistent with our previous observations with native UvrB that introduction of a bubble structure to the DNA substrates at the adduct could abolish the requirement for UvrA for efficient substrate incisions by $\operatorname{UvrBC}(5,6)$. The UvrA is indispensably required for the activity of strand opening of nonbubble normal substrate carried out by the $\mathrm{UvrA}_{2} \mathrm{~B}$. However, the incisions were adductspecific as the bubble DNA without an adduct was not a substrate for incisions in either the presence or absence of UvrA. These implied that UvrB could bind to damaged DNA bubble substrate although the native UvrB binding was not stable enough to be resolved in the gel mobility shift assay. It is most likely that the replacement of tyrosine at residue 95 with a tryptophan of UvrB essentially increases the stability of the intermediate formation of UvrBDNA.

Binding of $U V r A$ and $U v r B(Y 95 W)$ to Adducts. Although $\mathrm{UvrB}(\mathrm{Y} 95 \mathrm{~W})$ by itself efficiently recognized the structurally specific DNA substrates, how the mutant protein recognizes DNA damage under normal conditions in the presence of UvrA is a question that remains to be addressed. To examine the activity of the mutant proteins during their interactions with UvrA, strand opening, and thus the subsequent loading by UvrA to the damaged site, we performed gel mobility shift assays for the interaction of UvrA and UvrB (either wild type or mutants) 
with a normal nonbubble DNA substrate in the presence of ATP (Figure 3C). Consistent with the binding results described above, the data in Figure 3C clearly show that UvrB(Y95W) was the most efficient protein to form UvrB-DNA complex. Formation of this complex is the result of a series of sequential steps that include the two steps of damage recognition and DNA unwinding by the helicase activity of UvrB upon its interaction with UvrA, as well as the dissociation of UvrA. The consistent formation of the UvrB(Y95W)-DNA complex in the presence and absence of UvrA with the nonbubble and bubble substrates, respectively, suggests that the same complex is formed in the two cases.

Fluorescence Spectroscopic Determinations. By substituting the individual aromatic residues in the $\beta$-hairpin with a single tryptophan, we have been able to render the UvrB protein highly fluorescent at specific sites (the native protein contains no tryptophan). Examination of the fluorescent properties of these mutant proteins may provide important knowledge about the environment of specific residues in the hairpin. As shown in Figure 4, the tryptophan fluorescence was recorded for the three UvrB mutants: Y92W, Y95W, and Y101W. The $\gamma_{\max }$ of the emission spectra are 346, 351, and $356 \mathrm{~nm}$ for Y92W, Y101W, and Y95W, respectively. The apparent blue shifts of the spectra of Y92W from Y101W from Y95W indicated that, among the three residues, residue 92 is in the most hydrophobic environment followed by residue 101. In contrast, residue 95 seems to be in a fully polar environment as its $\gamma_{\max }$ is almost the same as that of free trypophan in the same buffer (16). Consistently, the order of emission intensities for the proteins was Y95W > Y101W. Y92W. The strikingly lower fluorescence intensity of $\mathrm{Y} 92 \mathrm{~W}$ relative to those of the other two residues is probably an indication that the Y92 residue is extensively involved in molecular interactions with some surrounding fluorescence quenching residues, which, as a result, efficiently quench the tryptophan fluorescence at residue 92. Similarly, the partial fluorescence quenching of residue 101 fluorescence as compared to the fluorescence of Y95W suggests that this residue may interact with its nearby residues to some extent.

Fluorescence measurements were also used to quantify the binding of UvrB(Y95W) to the fluorescein-30 bp bubble substrate at $37{ }^{\circ} \mathrm{C}$. Here, titration experiments were carried out by monitoring the quenching of fluorescein fluorescence at $520 \mathrm{~nm}$ with excitation at $492 \mathrm{~nm}$ upon binding of the protein. As shown in Figure 5, the nonlinear least-squares fitting of the binding isotherm from at least three independent titrations produced a dissociation constant of 88.4 (3.7 nM (17). This value is at least 50-fold smaller than that $(5 \mu \mathrm{M})$ of the binding of UvrB to the psoralen or cisplatin-modified ssDNA (16). The dissociation constant for the binding of wild-type UvrB to modified double-stranded DNA was generally considered to be high and unmeasurable, and this was also the case in the present study with our own substrates.

Binding of UvrB and Mutants to the DPC Adduct. In comparison with the normal DNA adduct, the DPC adduct has a much bulkier structure because of the presence of the cross-linked protein. It is of interest to gain information on the mechanism of damage recognition of NER by comparing the binding of UvrB mutants to the two different types of adducts. We therefore conducted binding assays of UvrB and its mutants with the DPC substrate. Interestingly, Figure 6 shows that the pattern of the binding of these proteins to DPC is exactly the same as the binding of the same proteins to the normal adducts. That is, only the Y95W mutant is capable of efficiently recognizing the DPC substrate, while the other mutants exhibit no or negligible binding affinities. This further confirms the unique role of the Y95 residue in damage recognition, in contrast to the other mutants studied.

Molecular Modeling of E. coli Native UvrB and UvrB-(Y95W) Mutant Structures. Although the structure of $E$. coli UvrB is not available, crystal structures of UvrB proteins from $B$. caldotenax and $T$. thermophilus have been reported previously by several laboratories (7-9). In an attempt to gain structural insights into the binding of UvrB and its mutant proteins from 
E. coli, we modeled the structures of the E. coli proteins based on the X-ray crystal structure of B. caldotenax $\mathrm{UvrB}(7,13)$. The $E$. coli and B. caldotenax proteins share about $59 \%$ identical sequences, which make our homology modeling reasonable. As shown in Figure 7, among all of the aromatic residues around the DNA binding cleft, the Y95 residue in the hairpin loop is the only one with the aromatic ring pointing directly into the center of the cleft in the modeled structure of wild type E. coli $\mathrm{UvrB}$ (this is also true in the B. caldotenax and T. thermophilus UvrB structures). This makes the residue fully available for direct interaction with DNA. This is consistent with the results from the fluorescence spectroscopic determination that Y95 is most red shifted and, thus, most exposed to the aqueous environment. On the other hand, although the predicted structures of $E$. coli $\mathrm{UvrB}$ and $\mathrm{UvrB}(\mathrm{Y} 95 \mathrm{~W})$ are very similar as a whole, some interesting difference has been noted at the site of residue 95 , which is that the orientations of the aromatic ring of the residues are different. Specifically, in comparison to the orientation of the aryl ring of wild-type Y95, the mutant Y95W aryl ring is rotated and becomes fully perpendicular to a virtual plane dividing the DNA binding cleft. Interestingly, residue 96 exhibits no effect on the aryl ring orientation of Y95 and is perpendicular to that of the Y95.

\section{DISCUSSION}

Understanding of the interactions of UvrB with DNA damage is important owing to the central role of UvrB in the damage recognition and incision in $E$. coli nucleotide excision repair. However, the damage recognition carried out by UvrB in the mechanism of $E$. coli NER depends on the action of the molecular matchmaker protein UvrA. The latter loads UvrB to the damaged site. In other words, wild-type UvrB lacks a direct affinity for damaged DNA without the involvement of UvrA. The coexistence of UvrA in the interaction greatly limits the investigation of UvrB interaction with DNA. Although efforts have been made to study the interaction of UvrB with ssDNA damage (16), limited results have been obtained due to the extremely low binding affinities. In the present study, we have substituted individual aromatic residues in the $\beta$-hairpin region of $\mathrm{UvrB}$ with tryptophan or phenylalanine for biochemical studies. Our results indicate that a single and only a single mutation at residue 95 from tyrosine to tryptophan made the protein fully capable of binding to the DNA adduct in a bubble structure even in the absence of UvrA. Consistently, more stable complexes are also formed between the $\mathrm{UvrB}(\mathrm{Y} 95 \mathrm{~W})$ and normal DNA substrates in the presence of UvrA, in the normal NER process (Figure 3C), indicating that the formation of the same type of UvrB-DNA intermediate complex occurs in both cases. This conclusion is further supported by the fact that this mutant protein is fully functional in DNA damage incisions. Our results pinpoint the unique role of Y95 in the direct interaction of UvrB with DNA adducts, while Y96 residue does not seem to play a significant role in this binding nor in the biochemical properties of the interaction in which the residue $\mathrm{Y} 95$ is involved.

The unique role of residue 95 is further supported by an analysis of the predicted structure of E. coli UvrB from homology modeling and our fluorescence spectroscopic studies. As shown in Figure 7, among all of the aromatic residues in the $\beta$-hairpin, the Y95 residue is the only one which points directly into the spacious cleft between domain 3 and the $\beta$-hairpin without any apparently significant interactions with nearby amino acid side chains. This cleft is believed to be the main site for DNA docking $(7,8)$. Replacement of Y95 with a tryptophan residue enhanced the binding capability of this residue to DNA most likely due to the increased hydrophobicity and a more favorable orientation of the aromatic ring for hydrophobic stacking. In contrast, other aromatic residues in the hairpin were either buried internally or involved in close interactions with neighboring residues. Our fluorescence spectroscopy results strongly suggest that residue 92 at the base of the hairpin extensively interacts with other residues. This conclusion is fully consistent with the structural model (Figure 7) showing that this residue is completely buried inside the protein and is close to the tryptophan fluorescence quenchers Ser91 andLys114 $(18,19)$ and the potential quenchers of Asp117, Glu118, and Asp120 (20). 
The Y93 residue is in a similar environment since it is surrounded by Ser91, Ser141, Ser143, and Lys67. Although, like Y95, residue Y96 lies in the cleft between domain 3 and the $\beta$ hairpin, its aryl ring is oriented along the backbone of the hairpin which is perpendicular to the Y95 aromatic ring. Similarly, although residue Y101 is at the tip of the hairpin, its fluorescence is partially quenched. The structure shown in Figure 7 clearly suggests that residue 101 lies in the gap between domain $1 \mathrm{~b}$ and the hairpin and that it directly interacts with hydrophobic phenylalanines 108 and 366 .

Hydrophobic interactions have been suggested to play a role in the damage recognition of UvrABC $(12,16,21)$. It is generally believed that tryptophan is the most hydrophobic residue among the 20 amino acids (22). The order of hydrophobicity for the aromatic residues is tryptophan > phenylalanine > tyrosine (22). In the general mechanism, UvrB forms a stable complex with adduct after being loaded by UvrA and the subsequent dissociation of UvrA. This complex formation is fully dependent on the conformational changes in both the UvrB protein and the DNA. For instance, DNA strand opening at the adduct occurs during the formation of the complex (6). In the case without the catalysis of UvrA, significant enhancement of some original specific interactions (such as those involving Y95) may be needed to overcome the energy barriers to induce complex formation. Our systems, which include mutation of Y95W and the structure-specific DNA substrates, most likely meet these requirements since the mutation significantly increases the hydrophobicity at residue 95 (22) which is directly involved in these interactions. In other words, substitution of Y95 with tryptophan enhances the original hydrophobic interactions at this specific site and is reflected in an enhanced damage recognition of UvrB. The high affinity of this enhanced interaction was quantitatively determined and was confirmed using the noninvasive approach of fluorescence spectroscopy $\left(K_{\mathrm{d}}\right) 88.4(3.7 \mathrm{nM})$. Taken together, our results suggest that the hydrophobicity at residue 95 plays a key role in the interaction of UvrB with DNA substrate and that the hydrophobic stacking may dominate these interactions. In the wide-type $E$. coli NER, the UvrA mediates the binding of native UvrB to the adducts probably by optimizing the molecular structures of both DNA and UvrB protein and, thus, by lowering the energy barriers for the binding.

It is also interesting to note that the Y95W mutant binds efficiently not only to the damaged DNA but also to nondamaged bubble DNA, although the affinity for the latter is lower. No binding was observed with nondamaged DNA without the bubble structure (data not shown). Therefore, besides the adducted base or nucleotide, unmodified bases of DNA are also likely to be involved in the interactions and contribute to damage recognition. A possible model for the interactions is that residue 95 hydrophobically stacks or intercalates between the modified and unmodified bases or between the adducted base and unmodified base. In the case of the CPD substrate which contains no exogenous hydrophobic adduct, the covalent cross-linking between the two intrinsic pyrimidines results in a large local DNA structural distortion including disruptions of DNA helix and base stacking. As a result, the largely distorted bases of the pyrimidine dimer out of the normal DNA structure might therefore be seen and recognized as an adduct by the UvrB protein. In addition, it should be noted that the more stable complex of the damaged DNA with $\mathrm{UvrB}(\mathrm{Y} 95 \mathrm{~W})$ than the one formed with the native UvrB did not result in an enhanced efficiency in incisions. This might be either due to the formation of intermediates that are not involved in the rate-limiting step in NER or because of a slight change of molecular interactions in the subsequent steps of UvrABC incision, albeit this formation is crucial to the success of damage recognition and incisions. While it is generally true that relatively higher rates are assumed to depend on the existence of more stable intermediates, exceptions do occur in some cases.

The observation that DPC is a substrate for NER is interesting and should be further examined to gain more insight into the mechanism of damage recognition. As demonstrated in this study, 
like other types of DNA adducts that include BPDE, fluorescein, and CPD, the DPC substrate was also efficiently recognized by the $\mathrm{UvrB}(\mathrm{Y} 95 \mathrm{~W})$ protein. Two potential factors may play important roles in the DPC binding. First, considering the general flipping-out mechanism involved in the glycosylase-DNA damage interactions, a local DNA unwinding at the adduct was likely induced by the cross-linking of T4 pyrimidine dimer glycosylase (T4pdg) to the DNA although no structural data are available yet for the DPC adduct. Second, direct interactions may occur between the W95 residue of the UvrB mutant and hydrophobic or aromatic residues of T4-pdg. However, in contrast to the other types of DNA damage, it is the replacement of a tyrosine by tryptophan at residue 92 that significantly increases the UvrABC incision efficiency of DPC as compared with the efficiency associated with the native UvrB protein and other mutants. The significance of the effects of these residues on the recognition and incision appears to be different between the normal and DPC adducts. The discrepancy between the mutant UvrB binding and incision of DPC substrate is probably because, as discussed above, the formation of UvrB-DNA intermediate may not be a rate-limiting step in NER or because these aromatic residues may play different roles in the recognition of normal bulky and DPC adducts. Despite this, the broad range of the substrates for the binding by Y95W is consistent with the wide spectrum of substrates of NER. The fact that NER recognizes and removes the extremely bulky DPC adduct suggests that the DNA damage recognition of NER is relatively insensitive to the sizes of adducts as long as they are bulky enough to introduce helical distortion.

Finally, with the availability of the crystal structures of a couple of different bacterial UvrB proteins, the finding that $\mathrm{UvrB}(\mathrm{Y} 95 \mathrm{~W})$ is able to efficiently bind to DNA substrates provides a molecular model system for further mechanistic investigations of DNA damage recognition in NER. For instance, additional details about the damage recognition might be revealed by the studies using other biochemical methods or the X-ray cocrystallographic determinations.

\section{REFERENCES}

1. Van Houten B. Microbiol. Rev 1990;54:18-51. [PubMed: 2181258]

2. Sancar A. Annu. Rev. Biochem 1996;65:43-81. [PubMed: 8811174]

3. Wood RD. Biochimie 1999;81:39-44. [PubMed: 10214908]

4. Minko IG, Zou Y, Lloyd RS. Proc. Natl. Acad. Sci. U.S.A 2002;99:1905-1909. [PubMed: 11842222]

5. Zou Y, Luo C, Geacintov NE. Biochemistry 2001;40:2923-2931. [PubMed: 11258904]

6. Zou Y, Van Houten B. EMBO J 1999;18:4889-901. [PubMed: 10469667]

7. Theis K, Chen PJ, Skorvaga M, Van Houten B, Kisker C. EMBO J 1999;18:6899-6907. [PubMed: 10601012]

8. Machius M, Henry L, Palnitkar M, Deisenhofer J. Proc. Natl. Acad. Sci. U.S.A 1999;96:11717-11722. [PubMed: 10518516]

9. Nakagawa N, Sugahara M, Masui R, Kato R, Fukuyama K, Kuramitsu S. J. Biochem 1999;126:986990. [PubMed: 10578047]

10. Moolenaar FF, Hoglund L, Goosen N. EMBO J 2001;20:6140-6149. [PubMed: 11689453]

11. Skorvaga M, Theis K, Mandavilli BS, Kisker C, Van Houten B. J. Biol. Chem 2002;277:1553-1559. [PubMed: 11687584]

12. Zou Y, Liu TM, Geacintov NE, Van Houten B. Biochemistry 1995;34:13582-93. [PubMed: 7577947]

13. Sali A, Blundell TL. J. Mol. Biol 1993;234:779-815. [PubMed: 8254673]

14. Laskowski RA, MacArthur MW, Moss DS, Thornton JM. J. Appl. Crystallogr 1993;26:283-291.

15. Myles GM, Van Houten B, Sancar A. Nucleic Acids Res 1987;15:1227-1243. [PubMed: 3547334]

16. Hsu DS, Kim S-T, Sun Q, Sancar A. J. Biol. Chem 1995;270:8319-8327. [PubMed: 7713940]

17. Zou Y, Bassett H, Walker R, Bishop A, Amin S, Geacintov NE, Van Houten B. J. Mol. Biol 1998;281:107-119. [PubMed: 9680479]

18. Clark PL, Liu Z-P, Zhang J, Gierasch LM. Protein Sci 1996;5:1108-1117. [PubMed: 8762142] 
19. Lakowicz, JR. Principles of Fluorescence Spectroscopy. Kluwer Academic/Plenum Publishers; New York: 1999. 2nd ed.

20. Ladokhin, AS. Encyclopedia of Analytical Chemistry. Meyers, RA., editor. John Wiley \& Sons; Chichester: 2000. p. 5762-5779.

21. Van Houten B, Snowden A. BioEssays 1993;15:51-59. [PubMed: 8466476]

22. Wimley WC, White SH. Nat. Struct. Biol 1996;3:842-848. [PubMed: 8836100]

23. Miroux B, Walker JE. J. Mol. Biol 1996;260:289-298. [PubMed: 8757792] 


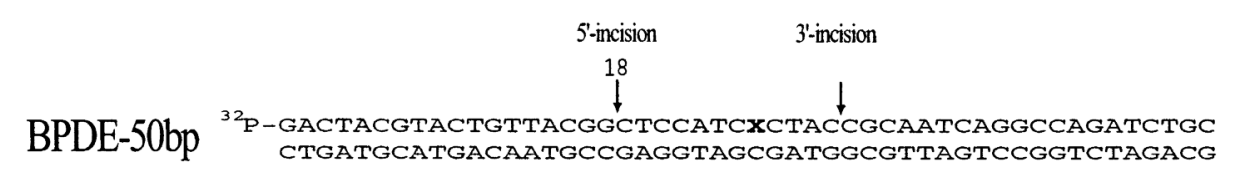

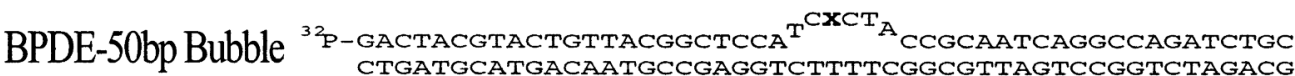 $\mathrm{X}=(+)$-cis-BPDE-N2-dG

\author{
X-30bp $\quad 32$ - -CCATCXCTACCGCAATCAGGCCAGATCTGC \\ GGTAGCGATGGCGTTAGTCCGGTCTAGACG

Figure 1:

Structures of DNA substrates used in the present study. The bold X in the sequences represents the single adducted nucleotide. The arrows indicate the major incision sites of UvrABC. 
A

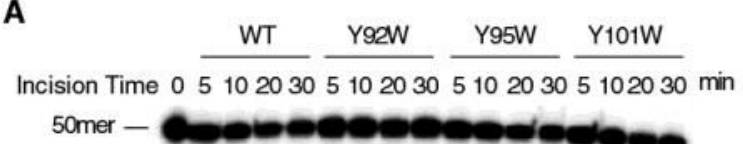

50mer -

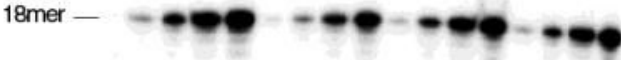

B

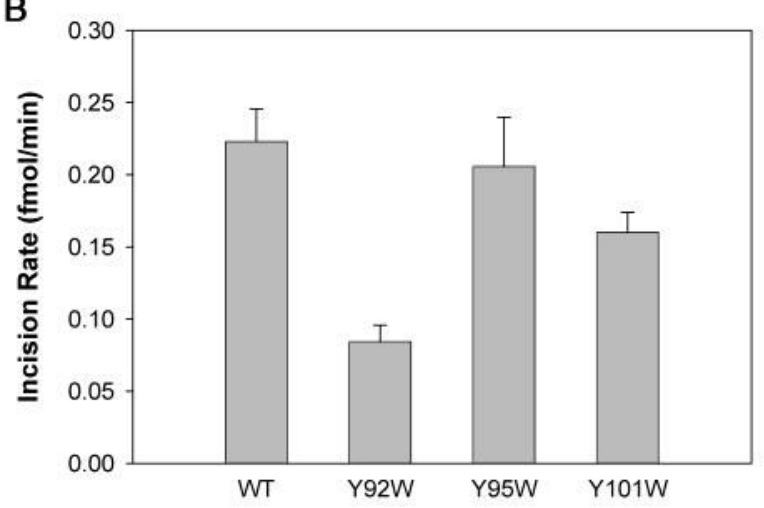

C

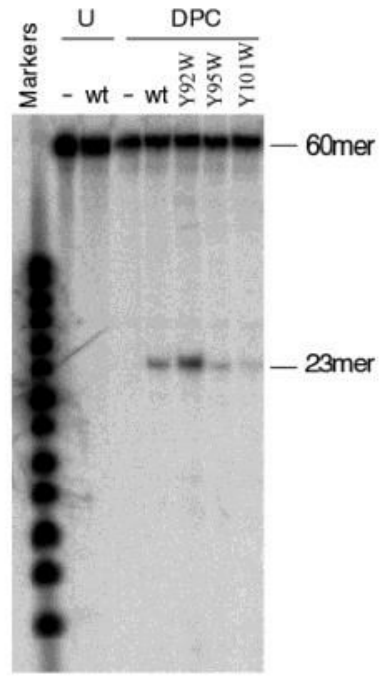

Figure 2:

Incision of BPDE and DPC substrates by UvrABC nuclease with wild-type (WT) or various mutant UvrBs. Panel A: 5'-Terminally labeled 50 bp substrates $(3 \mathrm{nM})$ containing a (+)-cisBPDE adduct were incubated with UvrABC (UvrA, $15 \mathrm{nM}$, UvrB or mutant, $250 \mathrm{nM}$, and UvrC, $50 \mathrm{nM}$ ) in the UvrABC buffer at $37{ }^{\circ} \mathrm{C}$ for the indicated time periods. The incision products were analyzed on a $12 \%$ polyacrylamide sequencing gel. The $50 \mathrm{mer}$ represents the intact top strand DNA (labeled), while the 18 mer represents the products of 5 '-incision. Panel B: Rates of UvrABC incisions of the substrate with wild-type UvrB and UvrB mutants. The error bars represent standard deviations of the measurements. Panel C: Double strand labeled substrates containing a DPC adduct were incised under the same experimental conditions except that the incubation time was $60 \mathrm{~min}$. The $60 \mathrm{mer}$ represents the intact bottom strand 
DNA which was also 5'-terminally labeled for the purpose of quantification, while the $23 \mathrm{mer}$ is the product of the $5^{\prime}$-incision. The $\mathrm{U}$ denotes the $60 \mathrm{bp}$ DNA containing a uracil instead of DPC as a control. 

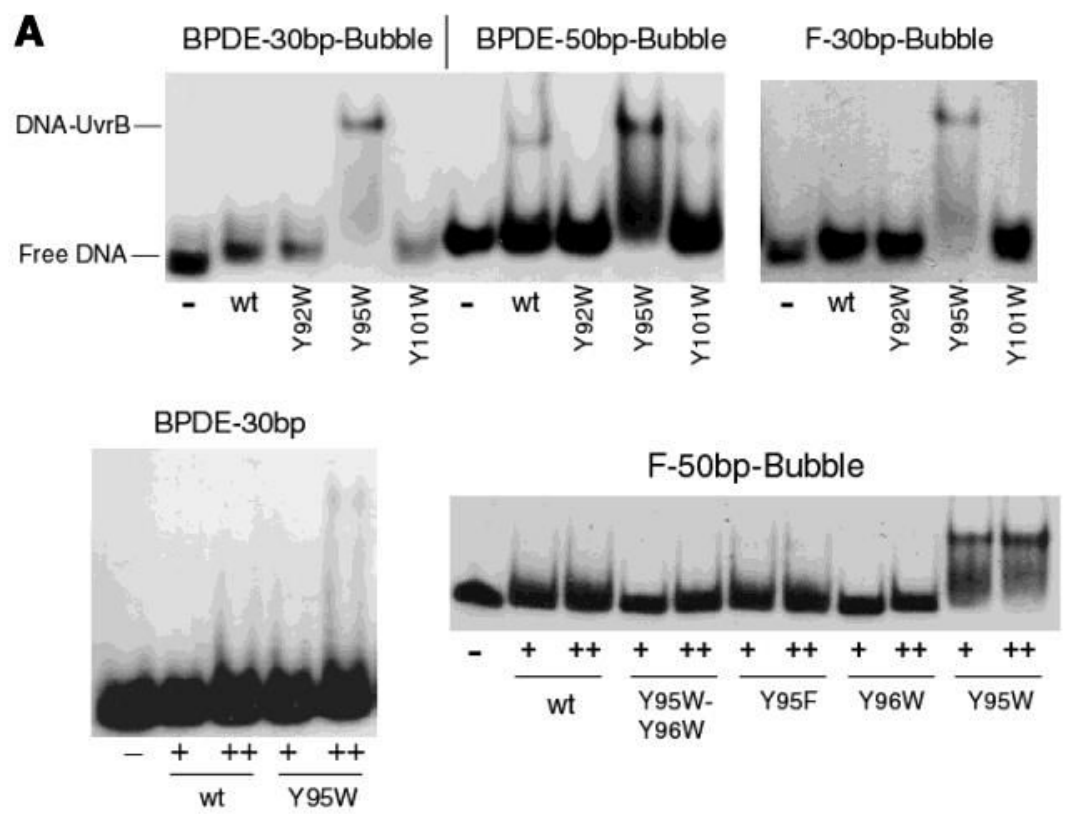

B

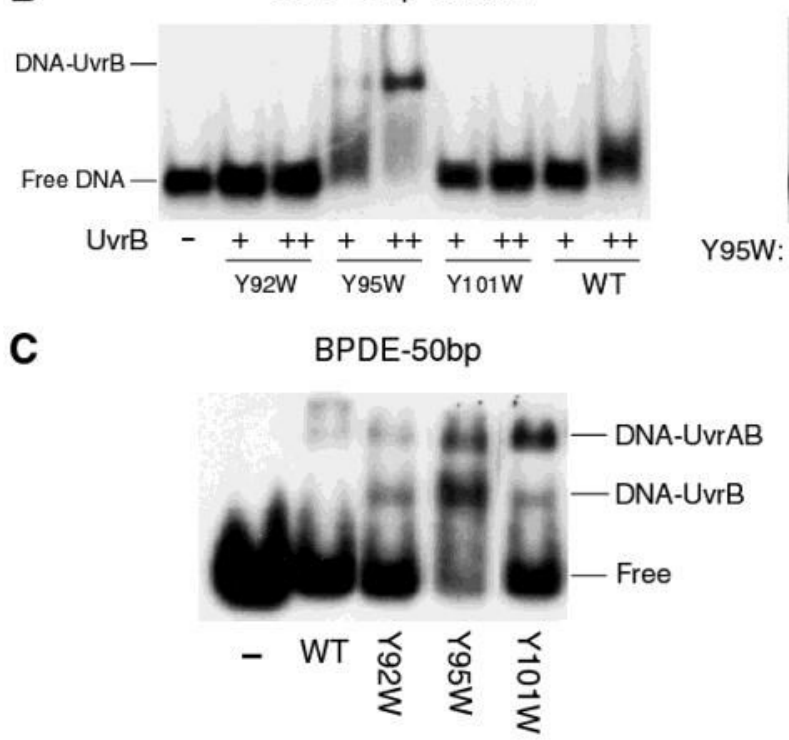

ND-30bp-Bubble

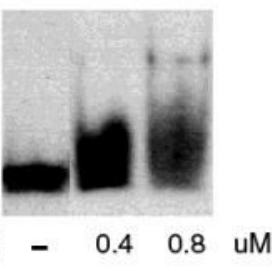

Figure 3:

Direct binding of UvrB and mutant proteins to various DNA substrates. Panel A: UvrB or UvrB mutants $(800 \mathrm{nM}$, or as indicated by,$+ 200 \mathrm{nM}$, and,$++ 600 \mathrm{nM})$ were incubated with DNA substrates $(3 \mathrm{nM})$ at $37^{\circ} \mathrm{C}$ for $15 \mathrm{~min}$ in the UvrABC buffer without ATP. The binding products were analyzed on a $3.5 \%$ native polyacrylamide gel. The notation free DNA represents the unbound DNA substrates, and DNA-UvrB represents the complexes formed between DNA and the UvrB mutants. The F-30bp-bubble substrate stands for fluorescein-30 bp bubble. Panel B: Same as in panel A except that the symbols + and ++ represent UvrB concentrations of 100 and $400 \mathrm{nM}$, respectively. Panel C: Labeled BPDE-DNA $50 \mathrm{bp}$ substrates were incubated with UvrA $(15 \mathrm{nM})$ and $\mathrm{UvrB} /$ mutants $(100 \mathrm{nM})$ at $37^{\circ} \mathrm{C}$ for $15 \mathrm{~min}$ in UvrABC buffer with $1 \mathrm{mM}$ ATP. The samples were analyzed on a $3.5 \%$ native polyacrylamide gel running with TBE buffer 
in the presence of $1 \mathrm{mM} \mathrm{ATP}$ and $10 \mathrm{mM} \mathrm{MgCl} 2 . \mathrm{F}, \mathrm{CPD}$, and ND stand for fluorescein, cyclobutane pyrimidine dimer, and nondamaged DNA, respectively. 


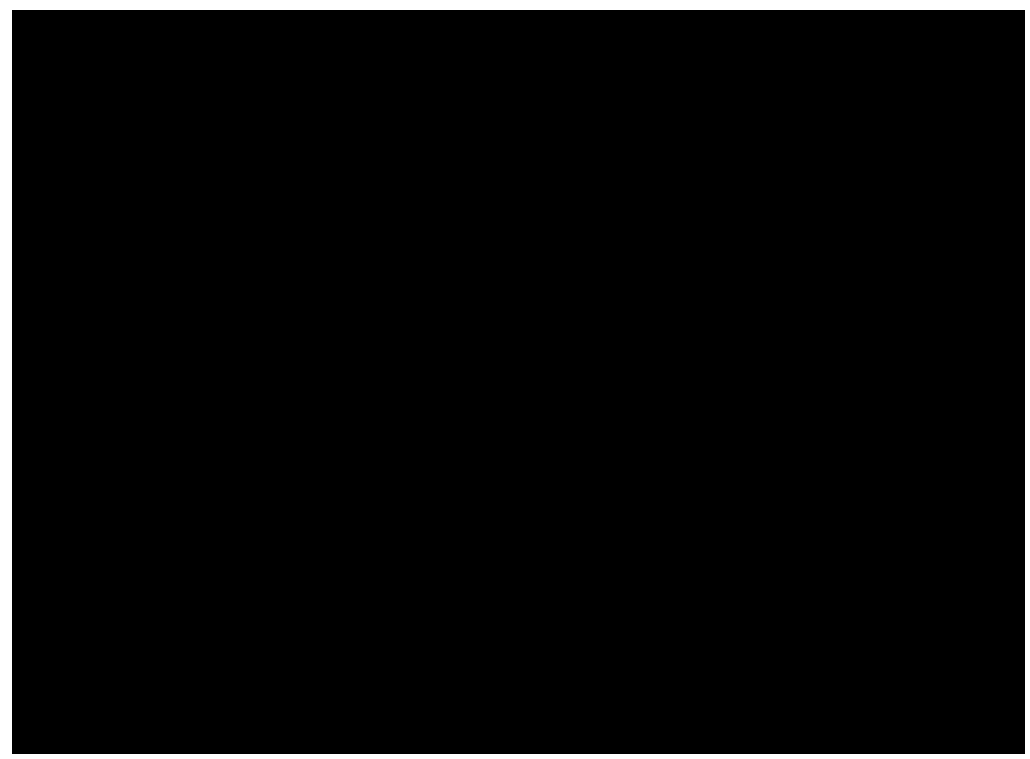

Figure 4:

Emission spectra of mutant UvrB proteins. The tryptophan fluorescence of the proteins (1 $\mu \mathrm{M}$ ) was recorded (in arbitrary units) at $25^{\circ} \mathrm{C}$ with excitation at $295 \mathrm{~nm}$ in $200 \mathrm{uL}$ of UvrABC buffer solution in the absence of ATP $(50 \mathrm{mM}$ Tris- $\mathrm{HCl}, \mathrm{pH} 7.5,50 \mathrm{mM} \mathrm{KCl}, 10 \mathrm{mM}$ $\mathrm{MgCl}_{2}, 5 \mathrm{mM}$ DTT). The slit widths were set at 3 and $5 \mathrm{~nm}$ for excitation and emission beams, respectively. 


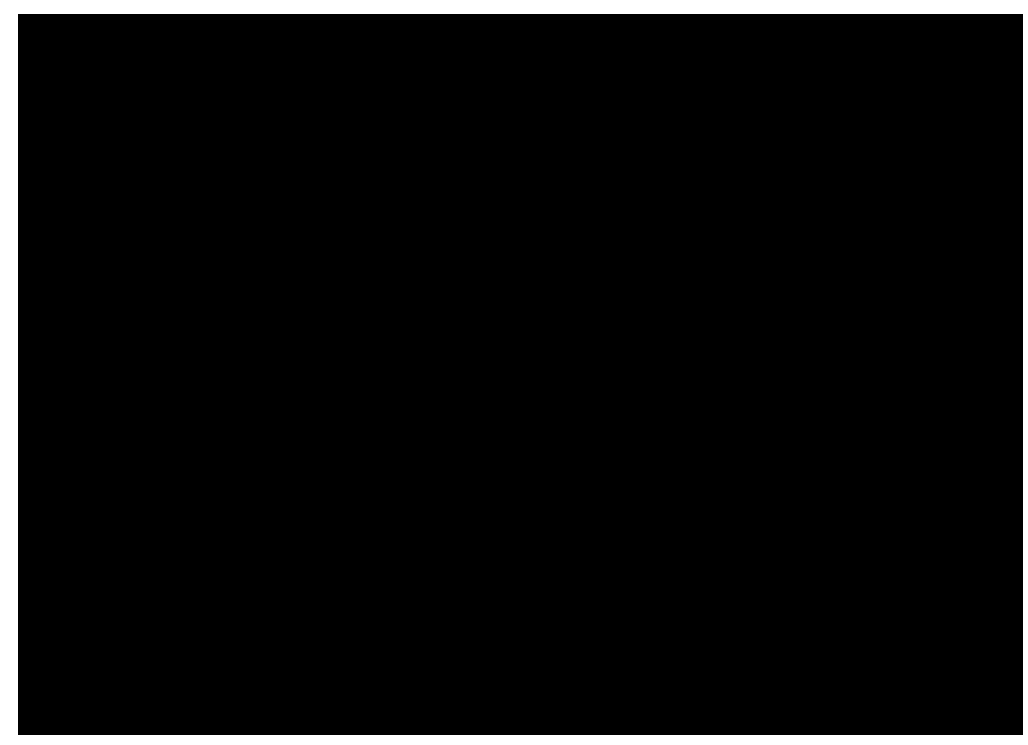

Figure 5:

Fluorescence titration of fluorescein-DNA substrate with UvrB(Y95W). Fluorescein-DNA substrate $(0.1 \mu \mathrm{M})$ was titrated with $\mathrm{UvrB}(\mathrm{Y} 95 \mathrm{~W})$ at $37^{\circ} \mathrm{C}$ in the $\mathrm{UvrABC}$ buffer without ATP by measuring the emission of fluorescein at $520 \mathrm{~nm}$ (excitation at $492 \mathrm{~nm}$ ). The slit widths were set at 1 and $5 \mathrm{~nm}$ for excitation and emission beams, respectively. 


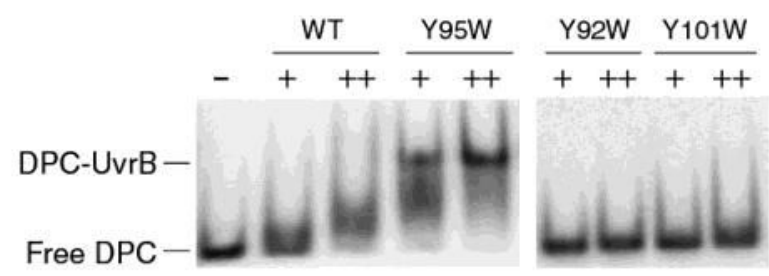

Figure 6:

Binding of UvrB and mutants to the DNA-protein cross-link adduct. UvrB or UvrB mutants of $400(+)$ or $800(++) \mathrm{nM}$ were incubated with DPC substrate $(3 \mathrm{nM})$ at $37{ }^{\circ} \mathrm{C}$ for $15 \mathrm{~min}$ in the UvrABC buffer without ATP. The binding products were analyzed on a $3.5 \%$ native polyacrylamide gel. Free DPC represents the unbound DPC-DNA substrate, and the DPCUvrB represents the complex formation between DNA and mutants. 
A

B

Figure 7:
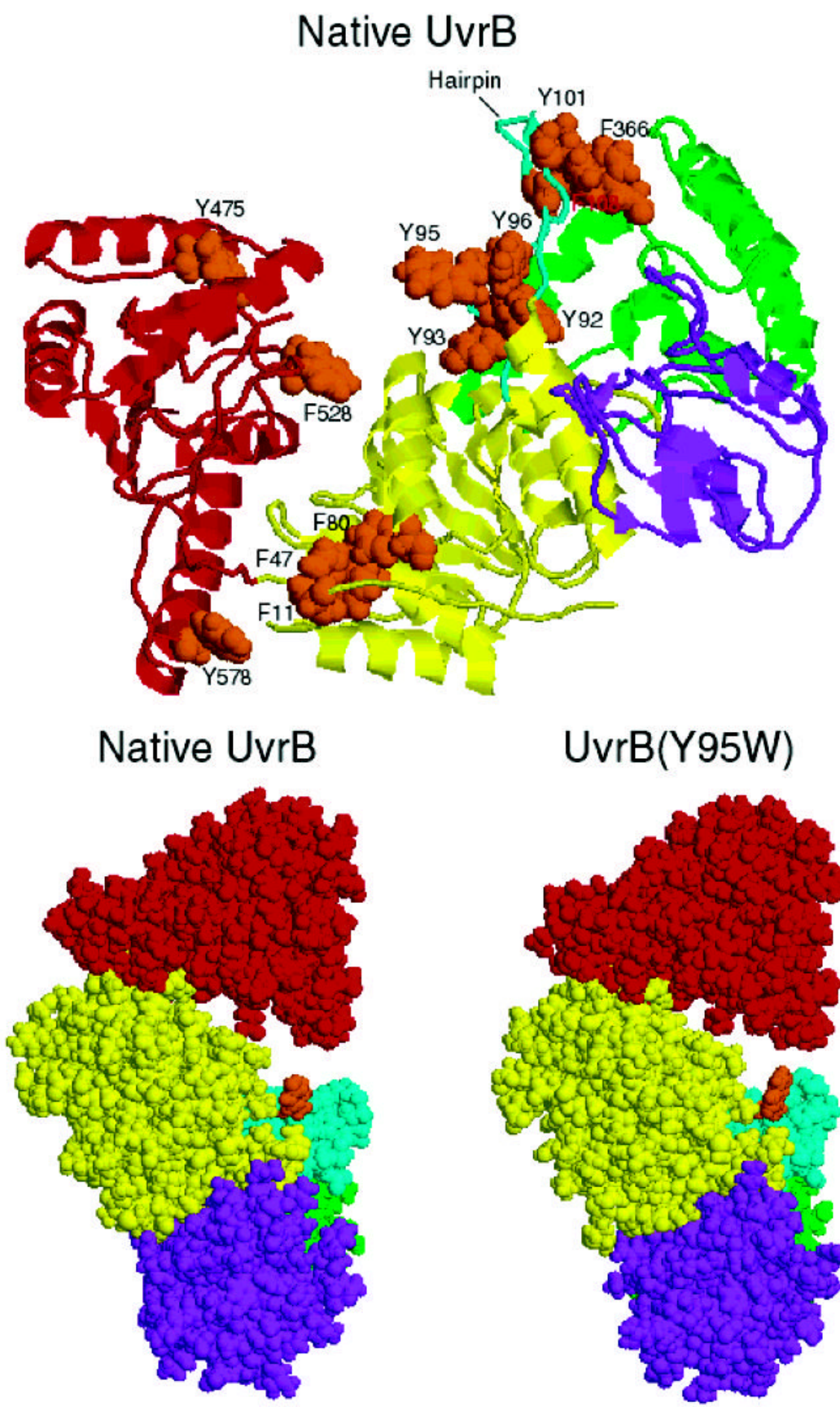

UvrB(Y95W)

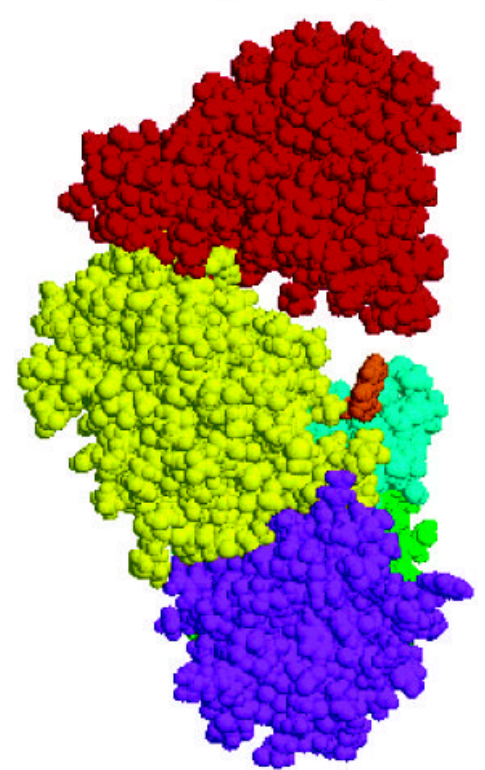

Modeled structures of E. coli UvrB and its mutant Y95W. Structures were generated by homology modeling based on the $2.6 \AA$ crystal structure of $B$. caldotenax $\mathrm{UvrB}$ (see Experimental Procedures). The quality of the modeled structures was evaluated by the PROCHECK program, indicating that about $98 \%$ of the residues are in the most favored and the additionally allowed regions of the Ramachandran plot. Domains of the structures are colored in yellow for domain 1a, green for domain 1b, purple for domain 2, red for domain 3, and cyan for the $\beta$-hairpin. Panel A shows the structure of native UvrB (ribbon). Aromatic residues (space-filling model) at the cleft are colored in gold. Panel B presents the space-filling 
structures of native $\mathrm{UvrB}$ and the $\mathrm{UvrB}(\mathrm{Y} 95 \mathrm{w})$ mutant for comparison mainly at residue 95. Y95 (native UvrB) and W95 (mutant) are colored in gold. 